\title{
A NOTE ON ENDOMORPHISM RINGS OF ABELIAN VARIETIES OVER FINITE FIELDS
}

\author{
By TETSUO NAKAMURA
}

Let $p$ be a prime and let $A$ be a simple abelian variety over a finite field $k$ with $p^{a}$ elements. In this note we ask some sufficient conditions that the endomorphism ring of $A$ over $k$ is maximal at $p$. Our result includes the first part of theorem 5.3 in Waterhouse [5]. The related facts should be referred to [5].

$\S 1$. Let $\operatorname{End}_{k}(A)$ be the ring of $k$-endomorphisms of a simple abelian variety $A$ over a finite field $k$ with $p^{a}$ elements. We shall always assume that $\operatorname{End}_{k}(A)$ is commutative. Then there exist a $C M$ field $E$ and an isomorphism $i_{A}: E \rightarrow$ $\operatorname{End}_{k}(A) \otimes \boldsymbol{Q}$. Let $R=\imath_{A}^{-1}\left(\operatorname{End}_{k}(A)\right)$ and let $K$ be the totally real subfield of index 2 in $E$. Let $f_{A}$ be the Frobenius endomorphism of $A$ over $k$ and put $\pi=\imath_{A}{ }^{-1}\left(f_{A}\right)$. Then $\pi$ is a Weil $p^{a}$-number, i. e. an algebraic integer such that $|\pi|^{2}=p^{a}$ in all embeddings of $E=\boldsymbol{Q}(\pi)$ into $\boldsymbol{C}$. Let $w$ be a place of $K$ above $p$ and $v$ be a place of $E$ with $v \mid w$. Then we have the following three cases;
(1) $v(\pi)=0$ or $v(\pi)=v\left(p^{a}\right)$.
(2) $v(\pi)=v\left(p^{a} \pi^{-1}\right)$.
(3) $v(\pi) \neq v\left(p^{a} \pi^{-1}\right)$ and $0<v(\pi)<v\left(p^{a}\right)$.

We call that $w$ is of type (1) (resp., (2), (3)) if $v$ satisfies (1) (resp., (2), (3)). This is independent of the choice of $v$ with $v \mid w$. Let $K_{w}$ be the completion of $K$ at $w$ and let

$$
\begin{aligned}
G_{w} & =\left(G_{1,0}\right)^{\left[K_{w}: \boldsymbol{\theta}_{p]}\right]}, \text { if } w \text { is of type (1), } \\
& =\left(G_{1,1}\right)^{\left[K_{w}: \boldsymbol{e}_{p]}\right]}, \text { if } w \text { is of type (2), } \\
& =G_{s, t}+G_{t, s}, \quad \text { if } w \text { is of type (3), }
\end{aligned}
$$

where $s=s(w)=\left[K_{w}: \boldsymbol{Q}_{p}\right] v(\pi) / v\left(p^{a}\right)$ and $t=t(w)=\left[K_{w}: \boldsymbol{Q}_{p}\right] v^{\prime}(\pi) / v^{\prime}\left(p^{a}\right)$ with the other place $v^{\prime}$ of $E$ above $w$. Then the formal group $\hat{A}$ of $A$ is isogenous to $\sum_{w \mid p} G_{w}$ (over the algebraic closure of $k$.) (cf. Manin [1], Chap. IV).

Now let $T_{p} A$ be the Dieudonné module of $\hat{A}$. Let $W=W(k)$ be the ring of Witt vectors over $k$ and $\sigma$ the automorphism of $W$ induced by the Frobenius

Received October 13, 1977. 
automorphism $x \rightarrow x^{p}$ of $k$. Let $\mathcal{A}=W[F, V]$ be the (non-commutative) ring defined by the relations $F V=V F=p, F \lambda=\lambda^{\sigma} F$ and $\lambda V=V \lambda^{\sigma}$ for $\lambda \in W$. Then $T_{p} A$ is a left $A$-module, $W$-free of $\operatorname{rank} 2 \operatorname{dim}(A)$. It is a well known result of Tate that

Assume further that

$$
\operatorname{End}_{k}(A) \otimes \boldsymbol{Z}_{p} \cong \operatorname{End}_{-l} T_{p} A .
$$

$\left(^{*}\right) R$ contains the maximal order $O_{K}$ of $K$.

Then as $T_{p} A$ is a module over $O_{K} \otimes \boldsymbol{Z}_{p}=\bigoplus_{w \mid p} O_{K}$, we have the corresponding decomposition $T_{p} A=\bigoplus_{w \mid p} T_{w}$, where $O_{K_{w}}$ is the ring of integers of $K_{w}$. We see that $T_{w}$ is a Dieudonné module whose corresponding formal group is isogenous to $G_{w}$.

§2. THEOREM 1. Let the notations be as in $\S 1$. We assume (*) and the follownng, for each $w$ of type (2), $K_{w}$ is an unramified extension over $\boldsymbol{Q}_{p}$ of odd degree and $F T_{w}=V T_{w}$, and for each $w$ of type (3), $F^{t(w)} T_{w} \subset V^{s(w)} T_{w}(\operatorname{say} s(w)$ $<t(w)$.). Then $R$ is maximal at p, i.e. $R \otimes \boldsymbol{Z}_{p}$ is the maximal order of $E \otimes \boldsymbol{Q}_{p}$.

Proof. Let $L$ be the quotient field of $W=W(k)$, i.e. $L$ is the unramified extension over $\boldsymbol{Q}_{p}$, of degree $a$. Put $\mathscr{B}=L \otimes_{W} \mathcal{A}=L[F, V]=L\left[F, F^{-1}\right]$. Let $\bigoplus_{v \mid p} E_{v}$ be the decomposition of $E_{p}=E \otimes \boldsymbol{Q}_{p}$ into fields. On $L \otimes_{\boldsymbol{Q}_{p}} E_{p}=\bigoplus_{v \mid p}\left(L \otimes_{\boldsymbol{Q}_{p}} E_{v}\right)$ we have $L$ acting by left multiplication and $E_{p}$ by right multiplication. Let $f_{v}$ be the residue degree of $E_{v} / \boldsymbol{Q}_{p}$. Put $g_{v}=\left(f_{v}, a\right)$. Then $L E_{v}$ has degree $a / g_{v}$ over $E_{v}$ and $L \otimes E_{v}$ is a sum of $g_{v}$ copies of the composite extension:

$$
\begin{gathered}
L \otimes E_{v} \cong L E_{v} \oplus \cdots \oplus L E_{v} . \\
\omega \otimes \beta \longrightarrow\left\langle\omega \beta, \omega^{\sigma} \beta, \cdots, \omega^{\sigma-1} \beta\right\rangle .
\end{gathered}
$$

We define the action of $\sigma$ on $L \otimes E_{v}$ by acting on the $L$-factor. Then for $\left\langle x_{1}, \cdots, x_{g_{v}}\right\rangle \in \oplus L E_{v}$, we have $\sigma\left\langle x_{1}, \cdots, x_{g_{v}}\right\rangle=\left\langle x_{2}, \cdots, x_{g_{v}}, \tau\left(x_{1}\right)\right\rangle$, where $\tau=\sigma^{g_{v}}$ is the Frobenius automorphism of $L E_{v} / E_{v}$. Now we can choose $u \in L \otimes E_{v}$ with $N_{L \otimes E_{v} / E_{v}}(u)=\pi$, where $N$ is the norm map. Define $F=u \sigma$. Then $F \lambda=\lambda^{\sigma} F$ for all $\lambda \in L$, and $F^{a}=\pi$. Thus we have constructed an operation of $\mathscr{B}$ on $L \otimes E_{v}$ and hence on $L \otimes E_{p}$. Then as a $\mathscr{B}$-module

$$
V_{p} A=T_{p} A \otimes_{W} L \cong L \otimes E_{p} .
$$

(For details of the above facts, see Chap. 5, [5].) As $T_{p} A$ is an $\mathcal{A}$-invariant lattice in $V_{p} A$, we may suppose that $T_{p} A$ is an $\mathcal{A}$-invariant lattice in $L \otimes E_{p}$. Then $T_{w}$ is a lattice in $L \otimes_{a_{p}} E_{w} \subset L \otimes E_{p}$, where $E_{w}=E \otimes_{K} K_{w}$. Let $R_{w}$ $=\operatorname{End}_{u^{4}}\left(T_{w}\right)$, then we clearly have

$$
R \otimes Z_{p}=\bigoplus_{w \backslash p} R_{w}
$$

Now we claim that each $R_{w}$ is the maximal order of $E_{w}$.

(i) The case that $w$ is of type (1). Then $w$ splits in $E / K$. Since $\pi-p^{a} \pi^{-1}$ 
is a unit, we see that $O_{K_{w}}[\pi]$ is maximal. As $R_{w} \supset O_{K_{w}}[\pi], R_{w}$ is maximal.

(ii) The case that $w$ is of type (3). Then $w$ also splits in $E / K$ into $v$ and $v^{\prime} ; L \otimes_{\mathbf{a}_{p}} E_{w}=\left(L \otimes_{\boldsymbol{a}_{p}} E_{v}\right) \oplus\left(L \otimes_{\mathbf{a}_{p}} E_{v^{\prime}}\right)$. Take $\alpha, \alpha^{\prime} \in L E_{v}$ such that $N_{L E_{v}}(\alpha)=\pi$ and $N_{L E_{v}}\left(\alpha^{\prime}\right)=p^{a} \pi^{-1}$. We can put $F=\left(\langle 1, \cdots, 1, \alpha\rangle+\left\langle 1, \cdots 1, \alpha^{\prime}\right\rangle\right) \sigma$ on $\left(L \otimes E_{v}\right)$ $\oplus\left(L \otimes E_{v^{\prime}}\right)$. Say $v(\pi)<v^{\prime}(\pi)=v\left(p^{a} \pi^{-1}\right)$, then $s=\left[K_{w}: Q_{p}\right] v(\pi) / v\left(p^{a}\right)$ and $t=$ $\left[K_{w}: Q_{p}\right] v^{\prime}(\pi) / v\left(p^{a}\right)$. Since $T_{w}$ is a $W \otimes O_{K_{w}}$-module, we have a decomposition $T_{w}={\underset{\imath=1}{g}}_{i=1}^{g} T_{\imath}$, corresponding to the decomposition $W \otimes_{z p} O_{K_{w}}=\oplus W O_{K_{w}}\left(g=g_{v}\right)$. As $T_{w} \otimes_{z_{p}} \boldsymbol{Q}_{p}=L \otimes_{\boldsymbol{a}_{p}} E_{w}$, we have $T_{\imath} \otimes_{z_{p}} \boldsymbol{Q}_{p} \cong L K_{w} \otimes_{k} E\left(\cong L E_{v} \oplus L E_{v^{\prime}}\right)$. Thus $T_{\imath}$ is a $W O_{K_{w}}$-free module of rank 2 and $W\left[F^{g}, V^{g}\right]$-invariant. As a $W O_{K_{w}}$-module it has a basis of the form $\left(\lambda^{n_{\imath}}, 0\right),\left(\mu_{\imath}, \lambda^{m_{i}}\right)$ with $\mu_{\imath}=0$ or $v\left(\mu_{\imath}\right)<n_{\imath}$, where $\lambda$ is a prime element of $O_{K_{w}}$. From the assumption we have that $V^{-s} F^{t} T_{w}=p^{-s} F^{s+t} T_{w}$ $\subset T_{w}$; hence for each $\imath, p^{-s} F^{s+t} T_{i} \subset T_{\imath}$. Now $p^{-s} F^{s+t}$ operates on $T_{\imath}$ by $\left(\delta, \delta^{\prime}\right) \tau^{h}$, where $\delta=\alpha \cdot \alpha^{-} \cdots \alpha^{\tau^{h-1}} / p^{s}, \delta^{\prime}=\alpha^{\prime} \cdot \alpha^{\prime} \cdots \alpha^{\prime-h-1} / p^{s}$ and $h=(s+t) / g$. Then $p^{-s} F^{s+t}$ $\left(\mu_{\imath}, \lambda^{m_{i}}\right)=\left(\delta \tau^{h}\left(\mu_{\imath}\right), \delta^{\prime} \lambda^{m_{i}}\right)=\xi\left(\lambda^{n_{\imath}}, 0\right)+\eta\left(\mu_{\imath}, \lambda^{m_{i}}\right)$ for some $\xi, \eta \in W O_{K_{w}}$; hence $\xi \lambda^{n_{\imath}}$ $=\delta \tau^{h}\left(\mu_{2}\right)-\delta^{\prime} \mu_{2}$. Now $v(\alpha)=v(\pi) /(a / g)=(g s v(p)) /(s+t)$, hence $v(\delta)=0$ and $v\left(\delta^{\prime}\right)>0$; this implies $\mu_{\imath}=0$. Thus each $T_{\imath}$ has a basis of the form $\left(\lambda^{n_{\imath}}, 0\right),\left(0, \lambda^{m_{\imath}}\right)$ over $W O_{K_{w}}$. This shows that $R_{w}$ is maximal.

(iii) The case that $w$ is of type (2). As $K_{w} / \boldsymbol{Q}_{p}$ is an unramified extension of odd degree and $\operatorname{End}_{k}(A)$ is commutative, $w$ does not split in $E / K$. Let $v$ be the place of $E$ above $w$. Suppose first that $E_{v}$ is unramified. As $2 v(\pi)=a, a$ is even and hence $g_{v}$ is also even. Now $F T_{w}=V T_{w}$ implies that $V^{-1} F T_{w}=p^{-1} F^{2} T_{w}$ $=T_{w}$ and so $p^{-(a / 2)} F^{a} T_{w}=T_{w}$. This shows that $R_{w} \exists p^{-(a / 2)} \pi$. Since $p^{-(a / 2)} \pi$ is a unit in $R_{w}$, there exists a unit $u_{1}$ in $W \otimes R_{w}$ with $N_{W \otimes R_{w} / R_{w}}\left(u_{1}\right)=p^{-(a / 2)} \pi$ (cf. Prop. 7.3 and the proof of theorem 7.4 in [5], p. 554.). Put $u_{2}=\langle 1, p, 1, p, \cdots, 1, p\rangle$ $\in L \otimes E_{v}$. Then $u_{2} \sigma\left(u_{2}\right)=p$ and $N_{L \otimes E_{v} / E_{v}}\left(u_{1} u_{2}\right)=\pi$. Now we can put $F=\left(u_{1} u_{2}\right) \sigma$. Since $T_{w}$ is $W \otimes R_{w}$-invariant, we have $u_{1} T_{w}=T_{w}$. As $W \otimes R_{w}$ is invariant under $\sigma$, we also have that $\sigma^{\jmath}\left(u_{1}\right) T_{w}=T_{w}\left(\jmath=1,2, \cdots\right.$.). As $g^{\prime}=g / 2$ is odd, we have

$$
p^{-\left(g^{\prime}-1\right) / 2} F^{g^{\prime}} T_{w}=F\left(p^{-1} F^{2}\right)^{\left(g^{\prime}-1\right) / 2} T_{w}=F T_{w} \subset T_{w} .
$$

It follows, by the definition of $u_{1}, u_{2}$ and $F$, that $u_{2} \sigma^{g^{\prime}}\left(T_{w}\right) \subset T_{w}$. As in case (ii) we have a decomposition $T_{w}=\bigoplus_{\imath} T_{\imath}$, corresponding to $W \otimes O_{K_{w}}=\oplus W O_{K_{w}}$. Here $T_{\imath}$ is invariant under $F^{g^{\prime}}$; hence $u_{2} \sigma^{g^{\prime}}\left(T_{\imath}\right) \subset T_{\imath}$. As a $W O_{K_{w}}$-module $T_{\imath}$ has a basis of the form $\left(p^{n_{\imath}}, 0\right),\left(\mu_{\imath}, p^{m_{\imath}}\right)$ with $\mu_{\imath}=0$ or $v\left(\mu_{\imath}\right)<n_{\imath} . \quad u_{2} \sigma^{g^{\prime}}$ operates on $T_{\imath}$ by

$$
u_{2} \sigma^{g^{\prime}}\left(x_{1}, x_{g^{\prime}+1}\right)=\left(x_{g^{\prime}+1}, \text { p } \tau\left(x_{1}\right)\right), \quad \text { for }\left(x_{1}, x_{g^{\prime}+1}\right) \in T_{2} .
$$

Then applying the same argument as in the proof of theorem 5.3 in [5], p. 548, we see that $\mu_{\imath}=0$; hence $T_{w}=\oplus T_{\imath}$ is invariant under the maximal order of $E_{v}$.

Suppose next $E_{v}$ is ramified over $K_{w}$. Choose an $\alpha \in L E_{v}$ with $N_{L E_{v} / E_{v}}(\alpha)=\pi$, then we can put $F=\langle 1, \cdots, 1, \alpha\rangle \sigma$. We extend $v$ to $L E_{v}$ naturally. As $g=g_{v}$ is odd, we have from the assumption

$$
p^{-(g-1) / 2} F^{g} T_{w}=F\left(p^{-1} F^{2}\right)^{(g-1) / 2} T_{w}=F T_{w} \subset T_{w} .
$$


As $F^{g}=\langle\alpha, \cdots, \alpha\rangle \sigma^{g}$ and $v(\alpha)=g$, we see that $p^{-(g-1) / 2} F^{g}=\langle\lambda, \cdots \lambda\rangle \sigma^{g}$, where $\lambda=p^{-(g-1) / 2} \alpha$ and $v(\lambda)=1$. Now decompose $T_{w}$ into $\oplus T_{\imath}$, corresponding to $W \otimes O_{K_{w}}$ $=\oplus W O_{K_{w}} . \quad T_{\imath}$ is invariant under $F^{g}$ and has a basis of the form $p^{n_{\imath}}, \mu_{\imath}+p^{m_{\imath}} c$ with $\mu_{i} \in W O_{K_{w}}, \mu_{i}=0$ or $w\left(\mu_{\imath}\right)<n_{\imath}$, where $c$ is a prime element of $E_{v}$. Then we can also apply the argument in the proof of theorem 5.3 in [5] and we see that $T_{w}$ is invariant under the maximal order of $E_{v}$. Therefore $R \otimes \boldsymbol{Z}_{p}=\oplus R_{w}$ is maximal and the proof is completed.

$R E M A R K$. If $R_{w}=\operatorname{End}_{\mathfrak{A}}\left(T_{w}\right)$ is maximal, we can write out the condition of a base of $T_{w}$ (cf. p. 545 in [5]). Hence if $R_{w}$ is maximal for a place $w$ of $K$, of type (3), it is easy to show, by a direct calculation, that $F^{t(w)} T_{w} \subset V^{s(w)} T_{w}$.

Corollary. Let $\alpha_{p}=\operatorname{Spec} k[x] /\left(x^{p}\right)$ be as in [2], I.2-11. Assume that $\hat{A}$ is usogenous to $\left(G_{1,0}\right)^{m}+\left(G_{1,1}\right)^{n}$ for some $m, n$ and $a(A)\left(=\operatorname{dim}_{k} \operatorname{Hom}\left(\alpha_{p}, A\right)\right)=n$. Assume further $(*)$ and that for each place $w$ of $K$ of type (2), $K_{w}$ is an unramified extension of odd degree over $\boldsymbol{Q}_{p}$. Then $R$ is maximal at $p$. (For the property of $a(A), c f .[2],[3],[4]$.

Proof. Put $T=\Sigma T_{w}$, where the sum is taken over all $w$ of type (2). Since $a(A)=\operatorname{dim}_{k} T /(F, V) T$ and $n=\operatorname{dim}_{k} T / F T=\operatorname{dim}_{k} T / V T$, we have that $(F, V) T=F T$ $=V T$. Hence our conclusion is obvious by theorem 1 .

REMARK. This corollary is a result which includes the first part of theorem 5.3 in [5], p. 548 (a result due to Shimura); assume that $R\left(\cong \operatorname{End}_{k}(A)\right)$ is commutative and contains the maximal order of $K$. Assume also that $p$ splits completely in $K$. Then $R$ is maximal at $p$.

For, in this case, it is easy to see that $\hat{A} \sim\left(G_{1,0}\right)^{m}+\left(G_{1,1}\right)^{n}$ for some $m, n$, and, for each $w$ of type (2), $T_{w}=G_{1,1}$; hence $a\left(T_{w}\right)=1$ and therefore $a(A)=n$.

§3. LEMMA. Let $M$ be a finite extension of $\boldsymbol{Q}_{p}$ and $N$ be a quadratic extension of $M$. Let $O_{M}$ and $O_{N}$ be the maximal orders in $M$ and $N$, respectively, and $\lambda$ be a prime element of $O_{M}$. Let $R$ be an order in $O_{N}$ containing $O_{M}$. Then there exists a non-negative integer $n$ such that $R=O_{M}+\lambda^{n} O_{N}$.

Proof. Let $c$ be an element in $O_{N}$ such that $O_{N}=O_{M}[c]$. Then $R \cap c O_{M}$ $=c \lambda^{n} O_{M}$ for some $n \geqq 0$. We see that

$$
R=O_{M}+c \lambda^{n} O_{M}=O_{M}+\lambda^{n} O_{N} .
$$

Let $\pi$ be a Weil $p^{a}$-number such that its corresponding abelian varieties have commutative endomorphism rings and an isogeny type $\left(G_{1,0}\right)^{m}+\left(G_{1,1}\right)^{n},(n>0)$ for thier formal groups. Put $E=\boldsymbol{Q}(\pi)$ and let $K$ be the totally real subfield of $E$ of index 2. We assume that, for each place $w$ of $K$ of type (2), $K_{w} / \boldsymbol{Q}_{p}$ is unramified of odd degree. (cf. the corollary of theorem 1.)

Theorem 2. Let $\pi$ be as above. Assume, for each place $w$ of type (2), $w$ is ramified in E. Put $f_{w}=\left[K_{w}: \boldsymbol{Q}_{p}\right]$ and $g_{w}=\left(a, f_{w}\right)$. Let $R$ be an order in $O_{E}$ 
containing $O_{K}[\pi]$. Then $R$ is an endomorphism ring of an abelian varnety corresponding to $\pi$ if and only if, for each $w$ of type (2), $R_{w}$ contains $O_{K_{w}}+p^{r}{ }_{w} O_{E_{v}}$, where $v$ is the place of $E$ with $v \mid w$ and $r_{w}=\left(g_{w}-1\right) / 2$.

Proof. By Porism 4.3 in [5] we only need to consider the situation at $p$. We make $V=L \otimes_{\boldsymbol{a}_{p}} E_{p}$ a $\mathcal{B}$-module as in the proof of theorem 1. The condition

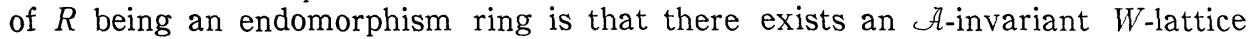
$T$ in $V$ such that $\operatorname{End}_{\lrcorner} T=R \otimes \boldsymbol{Z}_{p}$. Let $T$ be an $A$-invariant $W$-lattice in $V$ such that $\operatorname{End}_{\mathfrak{A}} T \supset O_{K}$. Then $T$ can be decomposed as $T=\bigoplus_{w \mid p} T_{w}$. (cf. $\S 1$ ) By the proof of theorem 1, End ${ }_{\mathcal{A}}\left(T_{w}\right)$ is maximal at each place $w$ of type (1). Next let $w$ be of type (2). Let $c$ be a prime element in $E_{v}$. Then $O_{E_{v}}=O_{K_{w}}[c]$. Let $\alpha$ be an element in $L E_{v}$ such that $N_{L E_{v} / E_{v}}(\alpha)=\pi$. Write $\alpha=d+b c$ with $b, d \in W O_{K_{w}}$. We see that $v(\alpha)=g_{w}=v(b)+1$ and $v(b)<v(d)$. Put $g=g_{w}$ and $r=r_{w}$. Then $v(b)=2 r$.

Put $F=\langle 1, \cdots, 1, \alpha\rangle \sigma$ on $L \otimes_{a_{p}} E_{w}$ with $E_{w}=K_{w} \otimes_{K} E=E_{v}$. We have a decomposition $T_{w}=\bigoplus_{\imath=1}^{g} T_{\imath}$, corresponding to the decomposition $W \otimes O_{K_{w}}=\oplus W O_{K_{w}}$. (cf. the proof of theorem 1) $T_{\imath}$ are $F^{g}$-invariant $W O_{K_{w}}$-lattice in $L E_{v}$. Then, for $x \in O_{E_{v}}$

$$
x \in \operatorname{End}_{\mathfrak{A}}\left(T_{w}\right) \Leftrightarrow x T_{i} \subset T_{\imath}, \quad \text { for all } \imath \text {. }
$$

Now write $\mathcal{E}\left(T_{\imath}\right)=\left\{x \in O_{E_{v}} \mid x T_{\imath} \subset T_{i}\right\}$. We may assume that $T_{\imath}$ has a basis $\left\{1, \mu+p^{m} c\right\}$, where $\mu=0$ or $v(\mu)<0\left(\mu \in L K_{w}\right)$. Write $c^{2}=h_{1} c+h_{2}$ with $h_{1}, h_{2} \in O_{K_{w}}$. Then $v\left(h_{1}\right) \geqq v\left(h_{2}\right)=2$.

We have

$$
\begin{aligned}
F^{g}\left(\mu+p^{m} c\right) & =(d+b c)\left(\mu^{\tau}+p^{m} c\right) \\
& =\left(d \mu^{\tau}+b p^{m} h_{2}\right)+\left(d p^{m}+b \mu^{\tau}+b p^{m} h_{1}\right) c \\
& =(\delta \mu+\eta)+\delta p^{m} c, \quad\left(\tau=\sigma^{g}\right) .
\end{aligned}
$$

for some $\delta, \eta \in W O_{K_{w}}$. Hence $\delta=d+b \mu^{\tau} p^{-m}+b h_{1}$ and $\delta \mu+\eta=d \mu^{\tau}+b p^{m} h_{2}$. If $\mu \neq 0$ and $v(\mu) \leqq 2 m$, then $v(\delta)=v(b)-2 m+v(\mu) \leqq v(b)$. Hence $v(\delta \mu)<\min \left\{v\left(d \mu^{\tau}\right), v\left(b p^{m} h_{2}\right)\right\}$. This shows that $\delta \mu$ is integral. Therefore we have $v(b) \geqq 2 m-2 v(\mu)$. If $v(\mu)>2 m$, then $v(\delta) \geqq v(b)+2$ and $v\left(b p^{m} h_{2}\right)<\min \left\{v(\delta \mu), v\left(d \mu^{\tau}\right)\right\}$. Therefore we have $v(b)$ $\geqq-2(m+1)$. If $\mu=0$, we also have $v(b) \geqq-2(m+1)$. On the other hand, we have the following; if $v(\mu) \leqq 2 m, \mathcal{E}\left(T_{\imath}\right)=O_{K_{w}}+p^{m-v(\mu)} O_{E_{v}}$ and if $v(\mu)>2 m$ or $\mu=0$, then $\mathcal{E}\left(T_{\imath}\right)=O_{K_{w}}+p^{-m-1} O_{E_{v}}$. As this will be proved by direct computation with almost the same argument as above, we omit its proof. Consequently, we have $\mathcal{E}\left(T_{2}\right)$ $\supset O_{K_{w}}+p^{r} O_{E_{v}}$. Hence $\operatorname{End}_{\mathfrak{l}}\left(T_{w}\right)=\bigcap_{i} \mathcal{E}\left(T_{\imath}\right) \supset O_{K_{w}}+p^{r} O_{E_{v}}$.

Now let $S=O_{K_{w}}+p^{t} O_{E_{v}}(t \leqq r)$ be an order in $O_{E_{v}}$ containing $O_{K_{w}}+p^{r} O_{E_{v}}$. Then $W S=W O_{K_{w}}+p^{t} W O_{E_{v}}$ in $L E_{v}$. Put $T_{r+1-s}=W O_{K_{w}}+p^{t-s} W O_{E_{v}}$ and $T_{r+1+s}=p^{s} T_{r+1-s}$ for $0 \leqq s \leqq r$. Here we consider that $T_{r+1-s}=W O_{E_{v}}$ if $t \leqq s$. Let $T=\bigoplus_{\imath=1}^{g} T_{\imath}$ in 
$L \otimes E_{w}=\oplus L E_{v} . \quad$ For $\left\langle x_{1}, x_{2}, \cdots, x_{g}\right\rangle \in T$ with $x_{i} \in T_{i}(\imath=1, \cdots, g)$, we have

and

$$
F\left\langle x_{1}, x_{2}, \cdots, x_{g}\right\rangle=\left\langle x_{2}, x_{3}, \cdots, x_{g}, \alpha x_{1}{ }^{\top}\right\rangle
$$

$$
V\left\langle x_{1}, x_{2}, \cdots, x_{g}\right\rangle=\left\langle p\left(\alpha^{-1} x_{g}\right)^{\tau^{-1}}, p x_{1}, \cdots, p x_{g-1}\right\rangle .
$$

Now we have the following relations;

$$
\begin{aligned}
& T_{1} \supset T_{2} \supset \cdots \supset T_{g-1} \supset T_{g} \supset \alpha T_{1}, \quad p T_{1} \subset T_{2}, \quad p T_{2} \subset T_{3}, \cdots, \\
& p T_{g} \subset \alpha T_{1}, \quad T_{1}=W O_{E_{v}} \text { and } T_{\imath}=T_{\imath} \text { for all } \imath .
\end{aligned}
$$

It is easy to see that $T$ is $\mathcal{A}$-invariant and $\operatorname{End}_{\mathcal{A}} T=S$. Our assertion now follows immediately from these facts.

Proposition 1. Let $\pi$ be as stated just before theorem 2. Let $A$ be an abelian variety corresponding to $\pi$ such that $R=\operatorname{End}_{k}(A)$ contains $O_{K}$. Let $w$ be of type (2) such that $w$ is unramified in $E$. Then the localization $R_{w}$ of $R$ at $w$ contams $O_{K_{w}}+p^{g-1} O_{E_{v}}$, where $g=\left(\left[K_{w}: Q_{p}\right], a\right)$.

Proof. Let $\langle\rho\rangle=\mathrm{Gal}\left(E_{v} / K_{w}\right)$ and $T=T_{p} A$. Let $T_{w}, T_{\imath}, \alpha, \mathcal{E}\left(T_{\imath}\right)$ be as in the proof of theorem 2. Then $R_{w}=\operatorname{End}_{\iota} T_{w} . T_{\imath}$ are $W\left[F^{g}, V^{g}\right]$-invariant, $W O_{K_{w}}{ }^{-}$ lattice in $L K_{w} \otimes_{K} E$. Let $\left(p^{n}, 0\right),\left(\mu, p^{m}\right)$ be a $W O_{K_{w}}$-basis of $T_{\imath}$, where $\mu=0$ or $v(\mu)<n$. $\quad \mu=0$ implies that $\mathcal{E}\left(T_{\imath}\right)$ is maximal. Suppose $\mu \neq 0$. We have

$$
\begin{aligned}
F^{g}\left(\mu, p^{m}\right) & =(1, \alpha) \tau\left(\mu, p^{m}\right)=\left(p^{m}, \mu^{-} \alpha\right) \\
& =\delta\left(p^{n}, 0\right)+\eta\left(\mu, p^{m}\right)=\left(\delta p^{n}+\eta \mu, \eta^{-} p^{m}\right)
\end{aligned}
$$

for some $\delta, \eta \in W O_{K_{w}} .\left(\tau=\sigma^{g}\right)$ Therefore $p^{m}=\delta p^{n}+p^{-m} \alpha^{\tau^{-1}} \mu^{2}$. If $n>m$, then $m$ $=-m+2 v(\mu)+v(\alpha)$. As $v(\alpha)=g$ is odd, we must have $n \leqq m$. Then $p^{m-n}=\delta$ $+p^{-m-n} \alpha^{--1} \mu^{2}$ shows that $v(\alpha) \geqq m+n-2 v(\mu)>n-v(\mu)$. On the other hand, for $x \in O_{E_{v}}$

$$
\begin{aligned}
x T_{\imath} \subset T_{\imath} \Leftrightarrow & \left(x \mu, x p^{m}\right)=\left(\delta p^{n}+\eta \mu, \eta^{-} p^{m}\right) \\
& \text { for some } \delta, \eta \in W O_{K_{w}} . \\
& \Leftrightarrow v\left(x-x^{\rho}\right) \geqq n-v(\mu) \\
& \Leftrightarrow x \in O_{K_{w}}+p^{n-v(\mu)} O_{E_{v}} .
\end{aligned}
$$

Therefore $\mathcal{E}\left(T_{\imath}\right)=O_{K_{w}}+p^{n-v(\mu)} O_{E_{v}} \supset O_{K_{w}}+p^{g-1} O_{E_{v}}$; as $R_{w}=\bigcap_{\imath} \mathcal{E}\left(T_{\imath}\right)$, this completes our proof.

COROllary. Let $\pi$ be as above. If, for each $w$ of type (2), a and $\left[K_{w}: \boldsymbol{Q}_{p}\right]$ are relatively prime, then $R=\operatorname{End}_{k}(A)$ containing $O_{K}$ is maximal at $p$.

This follows at once from theorem 2 and proposition 1. 
REMARK. This corollary also contains theorem 5.3 in [5]. For, in that case, $\left[K_{w}: \boldsymbol{Q}_{p}\right]=1$ for all $w$.

EXAMPLE. Let $\beta$ be a root of $f(x)=4 x^{4}+13 x^{3}-20 x-8=0 . \quad f(x)$ has four real roots in the interval $(-2 \sqrt{2}, 2 \sqrt{ } 2) .4^{3} f(x)=(4 x)^{4}+13(4 x)^{3}-20 \times 4^{2}(4 x)-8 \times 4^{3}$ shows that $f(x)$ has a root $\xi / 4$ in $\boldsymbol{Q}_{2}$ with a unit $\xi$ in $\boldsymbol{Q}_{2}$. Put $g(x)=f(x) /(4 x-\xi)$. Then $g(x) \in \boldsymbol{Z}_{2}[x]$ and $\left(1 / 2^{3}\right) g(2 x) \equiv x^{3}+x+1(\bmod 2)$. This shows that $g(x)$ is irreducible over $\boldsymbol{Q}_{2}$ and has a root in the cubic unramified extension of $\boldsymbol{Q}_{2}$. Since $f(x) \equiv 0(\bmod 7)$ has no root in $\boldsymbol{Z} / 7 \boldsymbol{Z}$, we see that $f(x)$ is irreducible over $\boldsymbol{Q}$. Therefore there are two places $w_{1}, w_{2}$ above 2 in $K=\boldsymbol{Q}(\beta)$ giving $w_{1}(\beta)=-2$ and $w_{2}(\beta)=1$. We have $K_{w_{1}}=\boldsymbol{Q}_{2}$ and $K_{w_{2}}$ is the cubic unramified extension of $\boldsymbol{Q}_{2}$. Let $\pi$ be a root of $x^{2}-4 \beta x+2^{5}=0 . \pi$ is a Weil $2^{5}$-number. $w_{1}$ splits in $E=\boldsymbol{Q}(\pi)$ and, since $(x / 4)^{2}-\beta(x / 4)+2$ is Eisenstein in $K_{w_{2}}, w_{2}$ is ramified in $E$. $\pi$ has a formal structure $G_{1,0}+\left(G_{1,1}\right)^{3}$ and a commutative endomorphism algebra. So $\pi$ satisfies the condition of the above corollary. Therefore an endomorphism ring containing $O_{K}$ is maximal at $p$.

For a supersingular abelian variety $A$ over $k$ (i. e. $\hat{A} \sim\left(G_{1,1}\right)^{m}$ with $m=\operatorname{dim}(A)$, cf. [4]), we have the following:

Proposition 2. Let a be even and put $a^{\prime}=a / 2$. Let $A$ be a simple supersingular abelian variety over $k$ such that $R\left(\cong \operatorname{End}_{k}(A)\right)$ is commutative. Assume that $F^{a^{\prime}} T_{p} A=V^{a^{\prime}} T_{p} A$. Then $R$ is maximal at $p$.

Proof. Let $\pi$ be the Weil number of $A$ over $k$. Then $\pi=p^{a} \zeta$, where $\zeta$ is a $n$-th root of 1 for some $n$. Since $V^{-a^{\prime}} F^{a^{\prime}}=p^{-a^{\prime}} F^{a}=p^{-a^{\prime}} \pi=\zeta$, we have $\zeta T_{p} A$ $=T_{p} A$. In $E \otimes \boldsymbol{Q}_{p}, \zeta \in E=Q(\pi)$ generates the maximal order over $\boldsymbol{Z}_{p}$. Therfore $R$ is maximal at $p$.

\section{REFERENCES}

[1] Manin, YU. I., The theory of commutative formal groups over fields of finite characteristic, Russian Math. Surv. 18 (6) (1963) 1-83.

[2] Oort, F., Commutative group schemes, Lect. N. Math. 15, Springer-Verlag (1965).

[3] OORT, F., Isogenies of formal groups, Proc. Konın. Neder. Akad. Wet. 78 (1975) 391-400.

[4] OORT, F., Which surfaces are prcducts of elliptic curves?, Math. Ann. 214 (1975) 35-47.

[5] Waterhouse, W.C., Abelian varieties over finite fields, Ann. scient. Éc. Norm. Sup. 2 (1969) 521-560.

Department of Mathematics,

College of General Education,

TôHOKU UNIVERsity 